\title{
IRREDUCIBILITY OF SOME REPRESENTATIONS OF THE GROUPS OF SYMPLECTOMORPHISMS AND CONTACTOMORPHISMS
}

\author{
ŁUKASZ GARNCAREK
}

\begin{abstract}
Aвstract. We show the irreducibility of some unitary representations of the group of symplectomorphisms and the group of contactomorphisms.
\end{abstract}

\section{INTRODUCTION}

Let $M$ be a smooth second-countable manifold. There exists a natural diffeomorphism-invariant measure class on $M$, consisting of measures having positive density with respect to the Lebesgue measure in every coordinate chart. We will refer to them simply as Lebesgue measures.

Let $\mu$ be a Lebesgue measure on $M$. For a group $G$ acting on $M$ by diffeomorphisms we may consider a series $\Pi_{\mu}^{\theta}$ of unitary representations on $L^{2}(M, \mu)$ given by

$$
\Pi_{\mu}^{\theta}(\gamma) f=f \circ \gamma^{-1}\left(\frac{d \gamma_{*} \mu}{d \mu}\right)^{1 / 2+i \theta}
$$

where $\theta \in \mathbb{R}$.

If a measure $v$ is equivalent to $\mu$, then the operator $T: L^{2}(M, \mu) \rightarrow$ $L^{2}(M, v)$ defined by

$$
T f=f\left(\frac{d \mu}{d v}\right)^{1 / 2+i \theta}
$$

gives an isomorphism of representations $\Pi_{\mu}^{\theta}$ and $\Pi_{v}^{\theta}$. In particular, if $\mu$ is equivalent to a $G$-invariant measure, the representations $\Pi_{\mu}^{\theta}$ are equivalent for all $\theta \in \mathbb{R}$.

For a diffeomorphism $\phi: M \rightarrow M$ we define its support supp $f$ as the closure of the set $\{p \in M: \phi(p) \neq p\}$. Compactly supported diffeomorphisms of $M$ form a group $\operatorname{Diff}_{c}(M)$. In [6] it was proved that for an infinite measure $\mu$ the representation $\Pi_{\mu}^{0}$ of the group $\operatorname{Diff}_{c}(M, \mu)$

2010 Mathematics Subject Classification. 57S20, 22A25. 
of compactly supported, measure-preserving diffeomorphisms of $M$ is irreducible. It follows that the representations $\Pi_{\mu}^{\theta}$ of the groups $\operatorname{Diff}_{c}(M, \mu)$ and $\operatorname{Diff}_{c}(M)$ are irreducible for any $\theta \in \mathbb{R}$. The idea of the proof is to take two functions $f, g \in L^{2}(M, \mu)$ and explicitly find a diffeomorphism $\phi$ such that $\left\langle f, \Pi_{\mu}^{0}(\phi) g\right\rangle \neq 0$, thus showing that $f$ and $g$ cannot lie in two distinct orthogonal invariant subspaces.

Representations of various subgroups of the group of diffeomorphisms are also studied in [4].

The purpose of this note is to present an enhancement of the argument from [6], and apply it to classical groups of diffeomorphisms: the group of symplectomorphisms and the group of contactomorphisms.

\section{Convolution on the Heisenberg group}

On $\mathbb{R}^{n}$ the following theorem holds (see Theorem 4.3.3 in [3] for a proof of a more general result):

Theorem 2.1. If $f, g \in L^{1}\left(\mathbb{R}^{n}\right)$ are compactly supported and nonzero, then $f * g$ is nonzero.

Proof. Let $\hat{h}(\xi)=\int h(x) e^{-i x \xi} d x$ denote the Fourier transform of $h \in$ $L^{1}\left(\mathbb{R}^{n}\right)$. Suppose that $f * g=0$. As $f$ and $g$ are compactly supported, their Fourier transforms extend to entire functions. Since $\hat{f} \hat{g}=\widehat{f * g}=$ 0 on $\mathbb{R}^{n}$, it follows by holomorphicity that $\hat{f} \hat{g}=0$ on $\mathbb{C}^{n}$, and either $\hat{f}$ or $\hat{g}$ must vanish. This contradicts the assumption that $f$ and $g$ are nonzero.

In this section we will prove an analogue of this theorem for squareintegrable functions on the Heisenberg group.

2.1. The Heisenberg group. Let $n$ be a positive integer. The multiplicative group of all matrices of the form

$$
\left(\begin{array}{ccc}
1 & \bar{x}^{T} & z \\
0 & I_{n} & \bar{y} \\
0 & 0 & 1
\end{array}\right),
$$

where $z \in \mathbb{R}, \bar{x}, \bar{y} \in \mathbb{R}^{n}$, and $I_{n}$ denotes the $n \times n$ identity matrix, is called the Heisenberg group $H_{n}$. It is a unimodular Lie group diffeomorphic with $\mathbb{R}^{2 n+1}$, and its Haar measure is the $(2 n+1)$-dimensional Lebesgue measure. We will identify $H_{n}$ with $\mathbb{R}^{2 n+1}$ as manifolds. The convolution of functions $f, g \in L^{1}\left(H_{n}\right)$ will be denoted $f *_{H} g$. 
2.2. Convolution of compactly supported functions on $H_{n}$. Let $f \in$ $L^{1}(\mathbb{R})$. Define

$$
T f(x)=\int_{-\infty}^{x} f(t) d t .
$$

If $f \in L^{2}(\mathbb{R})$ is supported in $[a, b]$, then it is integrable; furthermore, if $\int f(t) d t=0$, then supp $T f \subseteq[a, b]$ and we may write

$$
T f(x)=\int f(t) K_{[a, b]}(t, x) d t,
$$

where

$$
K_{[a, b]}(t, x)= \begin{cases}1 & \text { for } a \leq t \leq x \leq b, \\ 0 & \text { otherwise. }\end{cases}
$$

Hence, $T f \in L^{2}(\mathbb{R})$ and $\|T f\|_{2} \leq\left\|K_{[a, b]}\right\|_{2}\|f\|_{2}$, where $\left\|K_{[a, b]}\right\|_{2}$ stands for the $L^{2}$-norm of $K_{[a, b]} \in L^{2}\left(\mathbb{R}^{2}\right)$. We may iterate the process of applying $T$ to $f$ as long as it yields a function integrating to 0 . The next lemma shows that unless $f=0$, this process terminates.

Lemma 2.2. If $f \in L^{2}(\mathbb{R})$ is nonzero and compactly supported, then there exists $k \geq 0$ such that $T^{k} f \in L^{2}(\mathbb{R})$ and $\int T^{k} f(x) d x \neq 0$.

Proof. If there is no such $k$, then $T^{k} f \in L^{2}(\mathbb{R})$ and $\int T^{k} f(x) d x=0$ for all $k$. Suppose this is the case. We may assume that supp $f \subseteq[0,1]$, and replace $T$ with a bounded operator of the form (2.3) with kernel $K_{[0,1]}$.

Since $f$ is compactly supported, $\hat{f}$ extends to an entire function on C. We now have

$$
\widehat{T^{k} f}(\xi)=(i \xi)^{-k} \hat{f}(\xi),
$$

and by the Plancherel theorem

$$
4 \pi^{2}\left\|T^{k} f\right\|_{2}^{2}=\left\|\widehat{T^{k} f}\right\|_{2}^{2} \geq \int_{-1}^{1}|\hat{f}(\xi)|^{2} d \xi
$$

But $\|T\| \leq\left\|K_{[0,1]}\right\|_{2}<1$, so the left-hand side of the above inequality can be made arbitrarily small. Therefore $\hat{f}=0$, as it is an entire function vanishing on $[-1,1]$. This contradicts the assumption that $f$ is nonzero.

Let $f \in L^{2}\left(H_{n}\right)$ be compactly supported. Define $S f \in L^{1}\left(\mathbb{R}^{2 n}\right)$ by

$$
S f(\bar{x}, \bar{y})=\int_{\mathbb{R}} f(\bar{x}, \bar{y}, z) d z .
$$


If $S f=0$, we may also define $T f \in L^{2}\left(H_{n}\right)$ by

$$
T f(\bar{x}, \bar{y}, z)=\int_{-\infty}^{z} f(\bar{x}, \bar{y}, t) d t
$$

The proof of the next lemma consists of a straightforward application of the Fubini theorem:

Lemma 2.3. If $f, g \in L^{2}\left(H_{n}\right)$ are compactly supported, then

(1) $S\left(f *_{H} g\right)=S f * S g$,

(2) if $S f=0$, then $(T f) *_{H} g=T\left(f *_{H} g\right)$,

(3) if $S g=0$, then $f *_{H}(T g)=T\left(f *_{H} g\right)$.

Theorem 2.4. If $f, g \in L^{2}\left(H_{n}\right)$ are compactly supported and nonzero, then $f *_{H} g \neq 0$.

Proof. By Lemma2.2 there exist minimal $k$ and $l$ such that $S T^{k} f, S T^{l} g \in$ $L^{1}\left(\mathbb{R}^{2 n}\right)$ are nonzero and compactly supported. From Lemma 2.3 and Theorem 2.1 we obtain

$$
S T^{k+l}\left(f *_{H} g\right)=S\left(T^{k} f *_{H} T^{l} g\right)=S T^{k} f * S T^{l} g \neq 0,
$$

which implies that $f *_{H} g \neq 0$.

\section{Symplectic MANifolds}

3.1. Symplectic manifolds. Let $M$ be a symplectic manifold, that is a $2 n$-dimensional manifold equipped with a nondegenerate closed 2-form. A symplectomorphism of $(M, \omega)$ is a diffeomorphism $\phi \in$ $\operatorname{Diff}(M)$ satisfying $\phi^{*} \omega=\omega$. The group of all compactly supported symplectomorphisms will be denoted by $\operatorname{Sympl}_{c}(M, \omega)$. Since $\omega$ is nondegenerate, $\omega^{n}$ defines a positive measure $\mu$ on $M$, invariant under the action of $\operatorname{Sympl}_{c}(M, \omega)$.

A standard example of a symplectic manifold is $\mathbb{R}^{2 n}$ endowed with the symplectic form $\omega_{0}=\sum_{i=1}^{n} d x^{i} \wedge d y^{i}$. It is a theorem of Darboux that any symplectic manifold is locally symplectomorphic to $\left(\mathbb{R}^{2 n}, \omega_{0}\right)$ :

Theorem 3.1. For every $p \in M$ there exists a chart $\phi: U \rightarrow \mathbb{R}^{2 n}$ centered at $p$, such that $\left.\omega\right|_{U}=\phi^{*} \omega_{0}$.

Proof. See [1], Theorem 8.1.

The chart satisfying the conditions of Theorem 3.1 is called a Darboux chart. The pushforward of $\mu$ through a Darboux chart is the standard Lebesgue measure, up to a constant factor.

The flow $\mathrm{Fl}_{t}^{X}$ of a complete vector field $X \in \mathfrak{X}(M)$ consists of symplectomorphisms if and only if

$$
\mathcal{L}_{X} \omega=0 .
$$


There is an easy way to produce such vector fields. Namely, consider a compactly supported smooth function $f \in C^{\infty}(M)$. Since $\omega$ is nondegenerate, there exists a unique vector field $X_{f} \in \mathfrak{X}(M)$ such that $\left.X_{f}\right\lrcorner \omega=d f$, and it is not hard to show that this field satisfies (3.1).

For more information on symplectic manifolds see [1] and [5].

3.2. The representation $\Pi_{\mu}^{0}$ of $\operatorname{Sympl}_{c}(M, \omega)$. As $\mu$ is a $\operatorname{Sympl}_{c}(M, \omega)$ invariant measure, the only interesting representation is $\Pi_{\mu}^{0}$, taking the form

$$
\Pi_{\mu}^{0}(\gamma) f=f \circ \gamma^{-1} .
$$

Notice that the space of constant square-integrable functions is $\Pi_{\mu^{-}}^{0}$ invariant. It is nontrivial when $\mu(M)<\infty$. Let us denote its orthogonal complement by $\mathcal{H}$.

Theorem 3.2. The representation $\Pi_{\mu}^{0}$ of the group $\operatorname{Sympl}_{c}(M, \omega)$ on the space $\mathcal{H}$ is irreducible.

Lemma 3.3. Let $p \in M$ and let $\phi: U \rightarrow \mathbb{R}^{2 n}$ be a Darboux chart centered at $p$. Then there exist $r>0$ and for every $x \in B(0,2 r)$ a symplectomorphism $\tau_{x} \in \operatorname{Sympl}_{c}\left(U,\left.\omega\right|_{U}\right) \subseteq \operatorname{Sympl}_{c}(M, \omega)$ such that

(1) $\overline{B(0,3 r)} \subseteq \phi[U]$,

(2) $\phi \tau_{x} \phi^{-1}(y)=y+x$ for all $y \in B(0, r)$.

Proof. Take $r>0$ satisfying (1) and a bump function $h \in C^{\infty}\left(\mathbb{R}^{2 n}\right)$ supported in $\phi[U]$ and equal to 1 on $\overline{B(0,3 r)}$. On $\mathbb{R}^{2 n}$ there exists a linear function $f$ such that $X_{f}=x$ is a constant field. Then $X_{f h}=x$ on $\overline{B(0,3 r)}$ and supp $X_{f h} \subseteq \phi[U]$. The desired symplectomorphism is $\tau_{x}=\phi^{-1} \mathrm{Fl}_{1}^{X_{f h}} \phi$.

By using a standard argument we obtain the following well-known corollary:

Corollary 3.4. The action of $\operatorname{Sympl}_{c}(M, \omega)$ on $M$ is $k$-transitive for all $k \geq 1$.

Lemma 3.5. Let $\phi: U \rightarrow \mathbb{R}^{2 n}$ be a Darboux chart. Then for every nontrivial $\Pi_{\mu}^{0}$-invariant subspace $\mathcal{H}_{0}$ of $\mathcal{H}$, there exists $f \in \mathcal{H}_{0}$ such that $f \neq 0$ and supp $f \subseteq U$.

Proof. We may assume that $0 \in U=\phi[U] \subseteq \mathbb{R}^{2 n}$. Let $r>0$ be as in Lemma 3.3. Take a nonzero $g \in \mathcal{H}_{0}$. The 2-transitivity of $\operatorname{Sympl}_{c}(M, \omega)$ allows us to assume without loss of generality that there exists $c \in \mathbb{R}$ such that the sets $A=\{p \in B(0, r): \operatorname{Re} g(p)<c\}$ 
and $B=\{p \in B(0, r): \operatorname{Re} g(p)>c\}$ both have positive measure. By the Lebesgue density theorem there exist $a \in A$ and $b \in B$ with the property that $A$ (resp. $B$ ) has Lebesgue density 1 at $a$ (resp. $b$ ). Lemma 3.3 asserts the existence of a symplectomorphism $\tau=\tau_{b-a}$ that takes $a$ onto $b$ and preserves the Lebesgue density on $B(0,3 r)$. The function $f=g-\Pi_{\mu}^{0}(\tau) g \in \mathcal{H}_{0}$ then satisfies the conclusion of the lemma.

Proof of Theorem 3.2 Suppose that $\mathcal{H}=\mathcal{H}_{0} \oplus \mathcal{H}_{0}^{\perp}$ is a nontrivial decomposition into $\Pi_{\mu}^{0}$-invariant subspaces. Let $\phi: U \rightarrow \mathbb{R}^{2 n}$ be a Darboux chart, and let $r>0$ and $\tau_{x} \in \operatorname{Sympl}_{c}(M, \omega)$ be as in Lemma 3.3. Without loss of generality assume that $U=\phi[U] \subseteq \mathbb{R}^{2 n}$. By Lemma3.5 we may choose nonzero $f \in \mathcal{H}_{0}$ and $g \in \mathcal{H}_{0}^{\perp}$ supported in $B(0, r)$. We have

$$
\left\langle f, \Pi_{\mu}^{0}\left(\tau_{x}\right) g\right\rangle=\int_{B(0, r)} f(y) \overline{g\left(\tau_{x}^{-1}(y)\right)} d y=f * g^{*}(x),
$$

where $g^{*}(y)=\overline{g(-y)}$. But from Theorem 2.1 we know that this is nonzero for some $x \in \operatorname{supp} f * g^{*} \subseteq B(0,2 r)$. We obtain a contradiction, since $\Pi_{\mu}^{0}\left(\tau_{x}\right) g \in \mathcal{H}_{0}^{\perp}$.

\section{Contact manifolds}

4.1. Contact manifolds. Let $\operatorname{dim} M=2 n+1$. A contact form on $M$ is a 1 -form $\alpha \in \Omega^{1}(M)$ suct that $\alpha \wedge(d \alpha)^{n}$ is a volume form. Consider a $2 n$-dimensional distribution $\xi \leq T M$. There exists an open cover $\mathcal{U}=\left\{U_{i}\right\}$ of $M$, such that for every $\mathcal{U} \in \mathcal{U}$ the restriction $\left.\xi\right|_{U}$ is the kernel of a 1-form $\alpha_{U} \in \Omega^{1}(U)$. If the forms $\alpha_{U}$ are contact forms, we call $(M, \xi)$ a contact manifold. Unless $\xi$ is the kernel of a globally defined contact form, there is no distinguished measure on $M$.

Assume for the rest of this section that $(M, \xi)$ is a contact manifold. A contactomorphism of $(M, \xi)$ is a diffeomorphism $\phi \in \operatorname{Diff}(M)$, such that $\phi_{*} \xi=\xi$. The group of compactly supported contactomorphisms will be denoted by $\operatorname{Cont}_{c}(M, \xi)$.

An example of a contact manifold is the Heisenberg group $H_{n}$ with the distribution $\xi=\operatorname{ker} \alpha_{0}$, where $\alpha_{0}=d z-\sum_{i} y^{i} d x^{i}$ is a right-invariant form on $H_{n}$.

There is an analogue of Darboux theorem for contact manifolds:

Theorem 4.1. For every $p \in M$ there exists a chart $\phi: U \rightarrow H_{n}$ centered at $p$, such that $\left.\xi\right|_{U}=\operatorname{ker} \phi^{*} \alpha_{0}$.

Proof. See [2], Theorem 2.5.1. 
Let $U \subseteq M$ be such that $\left.\xi\right|_{U}=\operatorname{ker} \alpha$ for some $\alpha \in \Omega^{1}(U)$. There exists a unique vector field $R \in \mathfrak{X}(U)$ such that $\alpha(R)=1$ and $R\lrcorner d \alpha=0$, called the Reeb vector field. If $X \in \mathfrak{X}(U)$ is a complete vector field, then its flow $\mathrm{Fl}^{X}$ consists of contactomorphisms if and only if

$$
\mathcal{L}_{X} \alpha=u \alpha
$$

for some $u \in C^{\infty}(U)$. If we take any $f \in C^{\infty}(U)$, by nondegeneracy of $d \alpha$ there exists $X_{f} \in \mathfrak{X}(U)$ satisfying $\alpha\left(X_{f}\right)=f$ and $\left.X_{f}\right\lrcorner d \alpha=$ $d f(R) \alpha-d f$. These conditions imply equality (4.1). On the other hand, if $X$ satisfies (4.1), then it is of the form $X_{f}$ for $f=\alpha(X)$.

For more information on contact manifolds see [2].

\subsection{Representations of $\operatorname{Cont}_{c}(M, \xi)$.}

Lemma 4.2. Let $p \in M$ and let $\phi: U \rightarrow H_{n}$ be a Darboux chart centered at $p$. Then there exist an open set $V \subseteq H_{n}$, a convex open neighborhood $W$ of 0 in the Lie algebra of $H_{n}$, and for every $x \in \exp [W]$ a contactomorphism $\rho_{x} \in \operatorname{Cont}_{c}(U, \xi \mid u) \subseteq \operatorname{Cont}_{c}(M, \xi)$ such that

(1) $0 \in V \subseteq V V \subseteq \exp [W] \subseteq \overline{V \exp [W]} \subseteq \phi[U]$,

(2) $\phi \rho_{x} \phi^{-1}(y)=y x$ for all $y \in V$.

Proof. Existence of $V$ and $W$ satisfying (1) is obvious. Let $x=\exp v$, where $v \in W$. Then $v$ extends to a left-invariand vector field $X \in$ $\mathfrak{X}\left(H_{n}\right)$, and $\mathrm{Fl}_{t}^{X}=R_{\exp t v}$, where $R_{y}$ is the right multiplication by $y$. If $f=h \alpha_{0}(X)$, where $\left.h\right|_{V \exp [W]}=1$ and supp $h \subseteq \phi[U]$, then $X_{f}=X$ on $V \exp [W]$. The contactomorphism $\rho_{x}=\phi^{-1} \mathrm{Fl}_{1}^{X_{f}} \phi$ satisfies condition (2).

Corollary 4.3. The action of $\operatorname{Cont}_{c}(M, \xi)$ on $M$ is $k$-transitive for all $k \geq 1$.

Lemma 4.4. Let $\phi: U \rightarrow H_{n}$ be a Darboux chart. Then for every nontrivial $\Pi_{\mu}^{\theta}$-invariant $\mathcal{H}_{0} \leq L^{2}(M, \mu)$, there exists $f \in \mathcal{H}_{0}$ such that $f \neq 0$ and $\operatorname{supp} f \subseteq U$.

Proof. Without loss of generality assume that $0 \in U \subseteq H_{n}$ and $\left.\xi\right|_{U}=$ $\operatorname{ker} \alpha_{0}$. Let $\delta_{t}(\bar{x}, \bar{y}, z)=\left(e^{t} \bar{x}, e^{t} \bar{y}, e^{2 t} z\right)$ be the flow of the field $X=$ $(\bar{x}, \bar{y}, 2 z)$. We have $\delta_{t}^{*} \alpha_{0}=e^{2 t} \alpha_{0}$, so $X=X_{g}$ for some function $g \in$ $\mathrm{C}^{\infty}\left(H_{n}\right)$.

There exist $V=B(0, r) \subseteq \bar{V} \subseteq U$ and a function $h$ supported in $U$, such that $\left.h\right|_{V}=\left.g\right|_{V}$. Let $\psi_{t}=\mathrm{Fl}_{t}^{X_{h}}$. Then $\left.\psi_{t}\right|_{V}=\left.\delta_{t}\right|_{V}$ for $t<0$. Now, by transitivity of $\operatorname{Cont}_{c}(M, \xi)$, we may take a nonzero $f \in \mathcal{H}_{0}$ such that supp $f \cap V \neq \emptyset$. Since

$$
\int_{V}\left|\Pi_{\mu}^{\theta}\left(\psi_{t}\right) f\right|^{2} d \mu=\int_{\psi_{-t}[V]}|f|^{2} d \mu \underset{t \rightarrow \infty}{\longrightarrow} 0,
$$


there exists $t>0$ such that $f-\Pi_{\mu}^{\theta}\left(\psi_{t}\right) f$ satisfies the conclusion of the lemma.

Now, fix a Darboux chart $\phi: U \rightarrow H_{n}$ and a Lebesgue measure $\mu$ on $M$, such that $0 \in \phi[U]$ and $\phi_{*} \mu$ is the standard Lebesgue measure on $\phi[U] \subseteq \mathbb{R}^{2 n+1}$.

Theorem 4.5. For every $\theta \in \mathbb{R}$ the representation $\Pi_{\mu}^{\theta}$ of $\operatorname{Cont}_{c}(M, \xi)$ on the space $L^{2}(M, \mu)$ is irreducible.

Proof. The proof is analogous to the proof of Theorem 3.2 . Lemma 4.2 gives us $V \subseteq U$ and contactomorphisms $\rho_{x}$, such that for $f$ and $g$ supported in $V$ the matrix coefficient $\left\langle f, \Pi_{\mu}^{\theta}\left(\rho_{x}\right) g\right\rangle$ is nonzero for some $\rho_{x}$ because of Theorem 2.4 .

\section{RefERENCES}

[1] Ana Cannas da Silva. Lectures on Symplectic Geometry. Springer, 2001.

[2] Hansjörg Geiges. An Introduction to Contact Topology. Cambridge University Press, 2008.

[3] Lars Hörmander. The Analysis of Linear Partial Differential Operators I. SpringerVerlag, 1990.

[4] R. S. Ismagilov. Representations of Infinite-Dimensional Groups. American Mathematical Society, 1996.

[5] Dusa McDuff and Dietmar Salamon. Introduction to Symplectic Topology. Oxford University Press, 1998.

[6] A. M. Vershik, I. M. Gel'fand, and M. I. Graev. Representations of the group of diffeomorphisms. In Representation theory: selected papers. Cambridge University Press, 1982.

University of Wroceaw, Institute of Mathematics, Pl. Grunwaldzki 2/4, 50384 Wroceaw, Poland

E-mail address: Lukasz. Garncarek@math.uni . wroc.pl 